\title{
Irradiation Effects in MALDI, Ablation, Ion Production, and Surface Modifications. PART II: 2,5-Dihydroxybenzoic Acid Monocrystals
}

\author{
I. Fournier, ${ }^{*}$ C. Marinach, J. C. Tabet, and G. Bolbach \\ Laboratoire de Chimie Structurale Organique et Biologique, Université P and M Curie, Paris, France
}

\begin{abstract}
Irradiation effects at low and high laser fluence on 2,5-dihydroxybenzoic acid large crystals were investigated. Contrary to what was observed for matrices as cinnamic acid derivatives, no chemical degradation of matrix is evidenced and continuous ablation as well as ion production resulted of extended irradiation in all the fluence range corresponding to classical matrix-assisted laser desorption /ionization. Ripples are formed on the base of the crater for a limited number of laser shots under moderate fluence. For extended irradiation, conical shape craters are formed with the axis of the crater oriented along the incident direction of the laser beam. A study of the craters showed that ablation through the ablated volume slowly varied with the laser fluence when a strong increase of ion production (matrix and analyte) was recorded. Ablation volume was found to vary non-linearly with the number of laser shots. On a same spot, the ablated volume and the ion production were measured as a function of the laser energy. With an increasing laser energy (or fluence), the ablated volume slowly increases when the ion production strongly increases. This gives evidence of a decoupling between ablation and ionization. Interaction of the plume with the incoming beam is thus probable. (J Am Soc Mass Spectrom 2003, 14, 893-899) (C) 2003 American Society for Mass Spectrometry
\end{abstract}

$\mathrm{M}$ atrix-assisted laser desorption/ionization (MALDI) [1] is now a powerful method to produce ions of large molecules and its coupling with mass spectrometry (MS) provides a major analytical tool, particularly in proteomic studies and synthetic polymers characterization. To date, many of the methodologies developed for MALDI have been empirically determined. Indeed, the mechanisms involved in the ion production are very complex and cannot be experimentally studied in detail in all the time range of elementary processes. Briefly, these mechanisms involve ablation of molecules and aggregates from a solid state, the expansion of a plume from a very dense state to a very dilute gas. Ionization processes are likely involved in all the steps of the ablation and expansion. In addition, it is still difficult to predict for a small molecule absorbing at the laser wavelength whether it will be an effective or an inefficient matrix for the analysis of a given analyte.

Some recent reviews gave an overview of the com-

Published online June 25, 2003

Address reprint requests to Dr. G. Bolbach, Université P and M Curie, LCSOB, Bât F, Boîte 45, 4 Place Jussieu 75252 Paris Cedex 05, France. E-mail: bolbach@ccr.jussieu.fr

${ }^{*}$ Current address: Laboratoire de Neuroimunilogie des Annélidés, Université des Sciences et Technologies de Lille, 59655 Villeneuve d'Ascq Cedex, France. mon ideas on these ionization mechanisms [2, 3] involving specific chemical reaction, ion-molecule reaction [2] or proton transfer in aggregates $[3,4]$.

Generally, the mechanisms in MALDI are studied in two different ways. One deals with the ablation phenomenon mainly by molecular dynamics simulation [5, 6] at very short scale ( $\ll 1 \mathrm{~ns})$ and experimentally on the plume expansion [7] at medium and long-time scale (a few $\mu$ s to a few tens of $\mu \mathrm{s}$ ). The second deals with the ions produced by MALDI at the time scale of the ion time-of-flight, the role of the laser parameters [8], the target preparations and the nature of analyte ions. This subject occupies most publications in this field.

In this article, we will focus on the relationship between the ablated volume and the analyte ion production and on the irradiation effects using large 2,5dihydroxybenzoic acid single crystals doped with peptides. Classical MALDI targets, prepared from the socalled dried droplet method, are inefficient to use in studies of irradiation effects and the relationship between ablation (mainly neutral molecules and aggregates) and ionization because many small crystals are irradiated under the same laser spot.

In a recent article, we showed that irradiation effects of sinapinic acid large crystals, at the fluence close to the ion production threshold, were characterized by structural modifications associated with the disappearance 
of ion signals (matrix and analyte) [9]. These surface modifications are mainly due to decarboxylation of matrix molecules, and the vanishing of the ion production is likely associated to a decrease of the UV absorption of the target. This result explains why a limited number of laser shots are efficient to get ion signal in regular MALDI experiments using this matrix and the dried droplet method. However, these peculiar irradiation effects and the disappearance of the ion signal are troublesome when one wishes to study the ablation volume in connection with the ion production. Similar experiments were undertaken with another very popular matrix, i.e., 2,5-dihydroxybenzoic acid (2,5-DHB), for which ions are detected over a larger number of laser shots with a nearly constant yield. Previous investigations have compared the ablation of 2,5-DHB single crystals irradiated by UV and IR laser beams [10]. In UV, using a flat top laser and at fluence close to that of ion production, the irradiation by a few thousands laser shots gives rise to cone structures. In IR and at higher fluences, crater ablation without specific morphology was noted. In these experiments, using a gaussian laser beam profile, the ablation crater is roughly conic with its axis oriented in the laser direction [10].

The variation of the ablation volume, the ion production and the crater shape and volume as a function of both the laser energy or fluence and exposure are subjects of this article.

\section{Experimental}

\section{2,5-DHB Single Crystals}

2,5-DHB and peptides (ACTH 7-38 $\mathrm{mw}=3659.2$, bovine insulin $\mathrm{mw}=5733.5$ ) were purchased from $\mathrm{Al}$ drich (Lyon, France) and Sigma (Lyon, France), respectively, and they were used without purification. A saturated solution of 2,5-DHB $(400 \mu \mathrm{L})$ and a solution of peptide $(20 \mu \mathrm{L}$ with $20 \mathrm{pmol} / \mu \mathrm{L})$ were mixed in an Eppendorf tube. After vortexing (30 $\mathrm{min})$ and centrifugation $(15 \mathrm{~min})$, the supernatant $(\sim 300 \mu \mathrm{L})$ was removed and placed in a new tube. Without cover, the tube was kept at room temperature. A few days later, single crystals were formed on the walls. The larger crystals were chosen and dried on filter paper. Contrary to the sinapinic acid large crystal, no specific cleavage planes exist for the 2,5-DHB crystals with typical dimensions of $5 \times 0.15 \times 0.15 \mathrm{~mm}^{3}$ and thus, natural surfaces were used for this study. The longer dimension was in the [010] direction [11, 12]. It should be noted that the procedures published in the literature for obtaining these crystals $[12,13]$ were unsuccessful.

\section{MALDI Experiments}

MALDI experiments were performed on a Voyager Elite TOF mass spectrometer (PE Biosystems, Framingham, MA) in the linear mode and with delayed extraction. This instrument was equipped with a nitrogen laser (Laser Science, Franklin, MA) emitting at $337 \mathrm{~nm}$ and focused onto the target with an angle of incidence of $45^{\circ}$. The laser frequency was fixed to $2 \mathrm{~Hz}$. Ion focus along the drift tube was achieved by an ion wire guide. A video monitor allowed the display of a real-time video image of the sample spot during laser irradiation and following the macroscopic surface modifications through reflectance changes. The laser pulse energy, adjusted with a circular neutral filter, was measured using an energy meter (RJ7100 Laser Precision Corp).

\section{Crater Profiles and Desorbed Volume}

Ablation craters were analyzed by a profilemeter (Tancor P1). At least 10 profiles were recorded at step size of $10 \mu \mathrm{m}$ for each crater. The tip of the profilemeter $(5 \mu \mathrm{m}$ in diameter) allowed a resolution of $0.5 \mu \mathrm{m}$. Along a profile, the depth was recorded at steps of $0.2 \mu \mathrm{m}$. The data files were recorded, and a custom-made program (Visual Basic 5.0) allowed us to determine the area of a profile and to estimate the ablated volume. The ablated volume was calculated by considering that between two adjacent profiles with respective areas $S_{i}$ and $S_{i+1}$, the volume was $0.5 \times\left(\mathrm{S}_{\mathrm{i}}+\mathrm{S}_{\mathrm{i}+1}\right) \times \Delta \mathrm{y}, \Delta \mathrm{y}$ being the step length $(10 \mu \mathrm{m})$.

\section{Scanning Electron Microscopy}

The characterization of the irradiated samples and craters directly on the sample holder used for laser ablation was performed on a LEICA 440 scanning electron microscope without any manipulation of the sample. A gold layer of $150 \AA$ was deposited on the thickest crystals to avoid charging.

\section{Results}

\section{Irradiation Effects}

Contrary to what was observed for sinapinic acid single crystals [9], the matrix and analyte ion emission from the irradiated 2,5-DHB single crystals were found for a very large number of laser shots at the fluence threshold for ion production. In addition, the abundance of matrix ions $\left[(\mathrm{M}+\mathrm{H})^{+}, \mathrm{M}^{+\cdot}\right]$ and that of analyte $\left[(\mathrm{M}+\mathrm{H})^{+}\right]$ remain roughly proportional in a large exposure range (a few tens to a few thousands of laser shots). These features were noted for all the different irradiated surfaces oriented parallel or perpendicular to the axis [010] of the single crystal. After the irradiations, a crater is characteristic of the ablation with a depth increasing with the beam exposure. For fluence values higher than the fluence threshold, deeper craters were formed, as expected.

Figures $1 \mathrm{a}$ and $1 \mathrm{~b}$ show typical views of craters, using a light microscope, for different number of laser shots and different laser energy, respectively. The dimension of the craters was typically $(75 \times 90 \mu \mathrm{m})$ relatively independent of the laser energy and the shot number. 

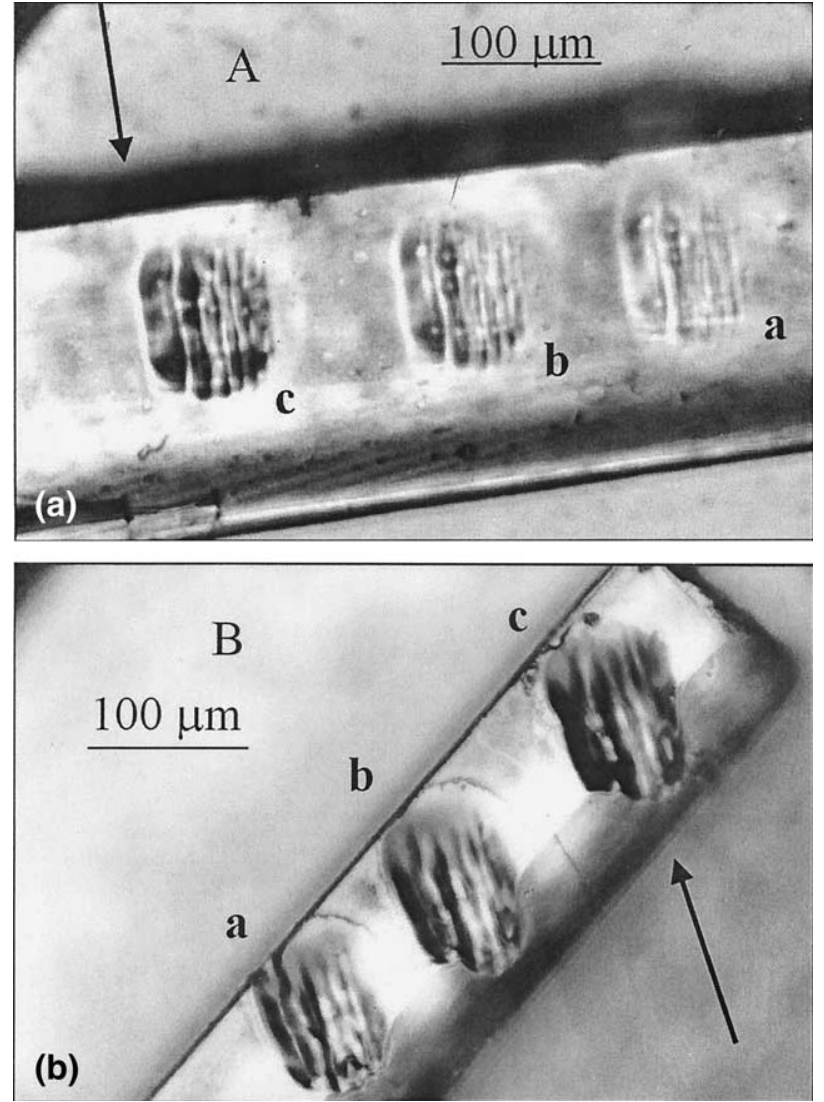

Figure 1. Light microscope images of a 2,5-DHB single crystal irradiated by laser shots: A. At laser energy $4.7 \mu \mathrm{J} /$ pulse with (a) 16 laser shots, (b) 32 laser shots, and (c) 64 laser shots. B. By 256 lasers shots at (a) $3.7 \mu \mathrm{J}$, (b) $4.1 \mu \mathrm{J}$, and (c) $4.7 \mu \mathrm{J}$. The arrow indicates the plane of the incident direction.

Interestingly, the base of these craters shows peculiar structures of alternative hill and valley as ripple with a typical periodic length of 5-10 $\mu \mathrm{m}$. These structures, as shown in Figure 1A and B, are parallel to the plane of the laser incidence and not to a crystallographic direction of the single crystal. In Figure 1A, the plane of the incident beam is perpendicular to the [010] direction of the single crystal and oriented by $45^{\circ}$ in Figure 1B.

For extended irradiation, the shape of the crater can be studied directly with a light microscope looking at a vertical section of the single crystal. As shown in Figure 2 , the shape of the crater was conical with a symmetry axis parallel to the incident direction of the laser beam. The depth of the crater along the incident direction $\left(45^{\circ}\right)$ was 115 and $140 \mu \mathrm{m}$ for 1536 and 2048 laser shots, respectively. At these strong irradiations, the structures of alternative hill and valley, mentioned above, completely disappear.

Detailed studies of the crater surface were performed using scanning electron microscopy (SEM). Figure 3 shows SEM images of the craters after extended irradiations at $2.5 \mu \mathrm{J} /$ pulse (below the threshold for ion production) after 2560 and 1760 laser shots. Similar dimensions were found for the crater $(60 \times 80 \mu \mathrm{m})$. Condensation products on the target surface were also

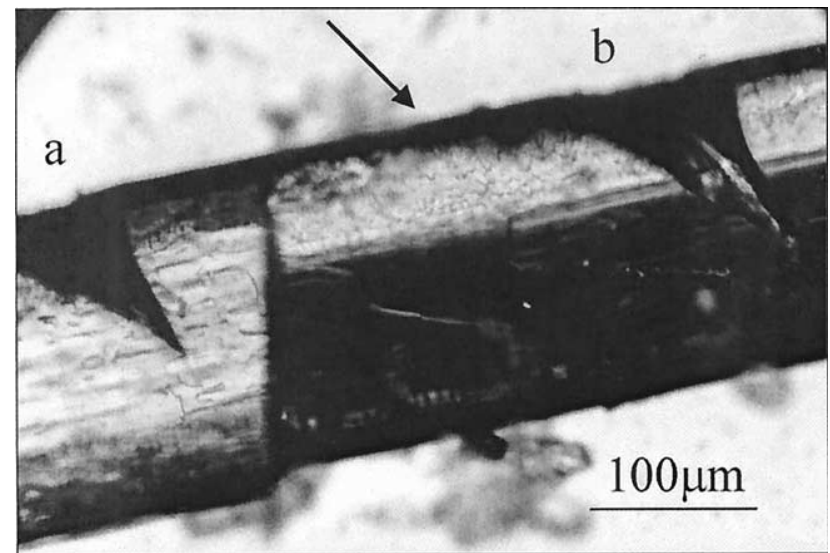

Figure 2. Light microscope images of two craters, seen along a vertical section of the single crystal, after irradiation by a $4.7 \mu \mathrm{J} /$ pulse for (a) 1536 laser shots and (b) 2048 laser shots. The arrow indicates the laser beam incident direction.

present all around the craters as previously noticed for sinapinic acid crystals [9]. In addition, no peculiar
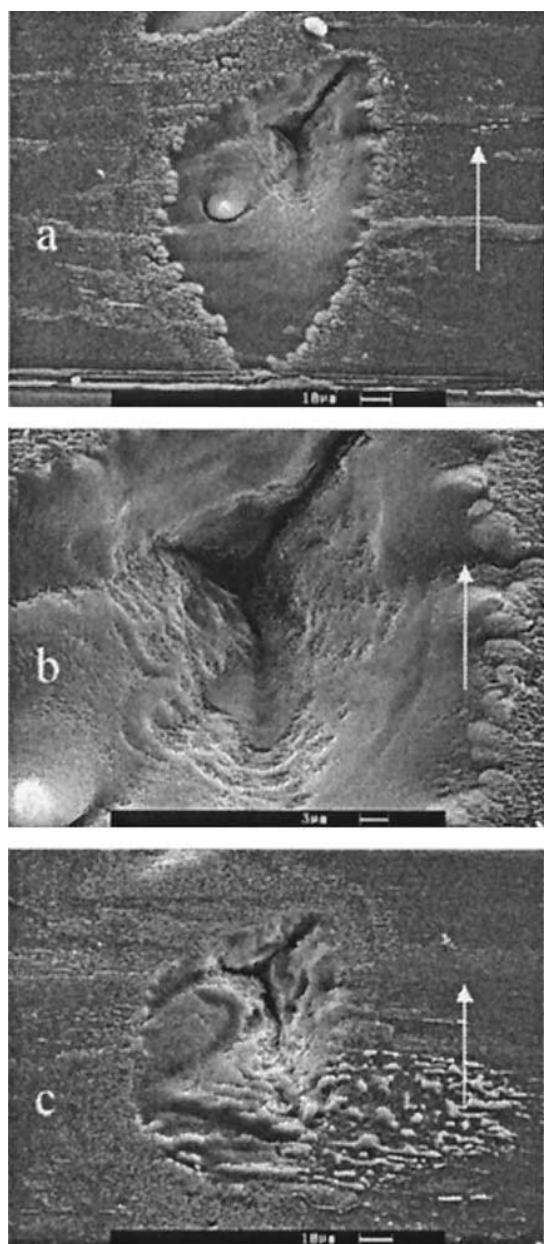

Figure 3. Scanning electron micrographs of 2,5-DHB single crystals irradiated by $2.5 \mu \mathrm{J} /$ pulse: (a) View of the crater after 2560 laser shots; (b) magnification of the base of the crater shown in (a); (c) crater induced by 1760 laser shots. The arrow indicates the plane of the incident direction. 


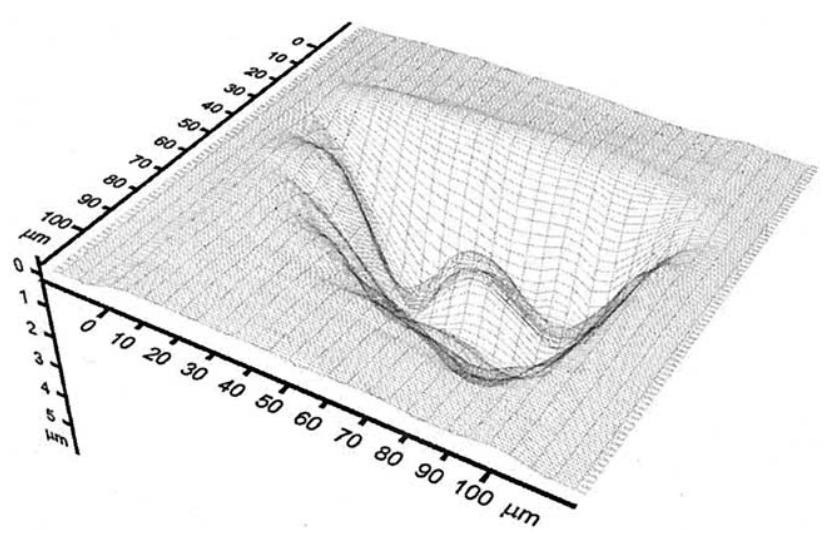

Figure 4. Crater in 2,5-DHB after 64 laser shots $(5.2 \mu \mathrm{J} /$ pulse $)$ rebuilt from the profilemeter data. Note the difference in the depth and lateral scales.

structure and morphology were distinguishable, and the surface of the craters appeared relatively smooth (Figure 3B), except a micro-conical structure (Figure 3A).

\section{Ablated Volume}

The ablated volume corresponding to an irradiation experiment was determined using a profile meter. Up to ten profiles, at a distance of $10 \mu \mathrm{m}$ apart, were performed to cover the entire crater. From these profiles, the volume was calculated and the crater rebuilt (Figure 4). One should note that this volume corresponds to both the volume ejected under vacuum and the volume of condensation found all around the crater. However, as condensation products are mainly deposited on the rim of the crater, and the profile depth was calculated relative to the rim, the estimated volume is thus that of material ejected under vacuum. The ablation volume was studied as a function of the exposure. As shown in Figure 5, the volume doesn't vary linearly with the number of laser shots, and the normalized volume relative to the shot number decreases with the shot number. The averaged volume per laser shot is typically $387 \mu \mathrm{m}^{3}$ and $126 \mu \mathrm{m}^{3}$ after irradiation with 32 and

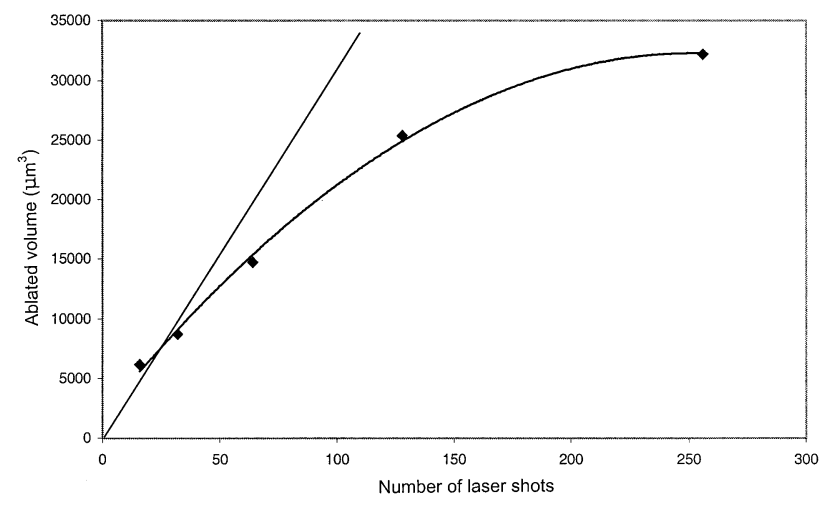

Figure 5. Variation of the ablated volume versus the number of laser pulse (laser energy $4.7 \mu \mathrm{J}$ ) for 2,5-DHB single crystals.

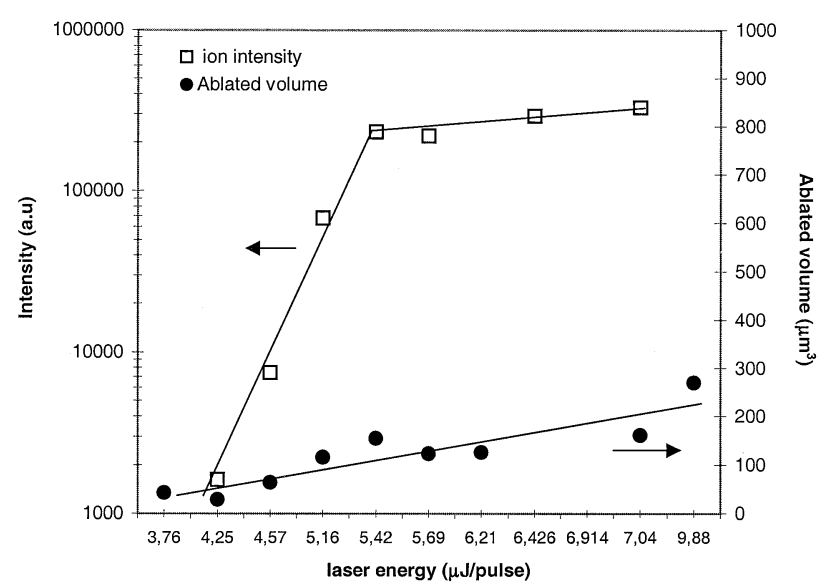

Figure 6. Ablated volume (right scale) and ion production (peak area protonated molecule of ACTH 7-38) (left scale) from 2,5-DHB single crystals irradiated by 256 laser shots as a function of the laser intensity. Two experiments were carried out at $5.4 \mu \mathrm{J} /$ pulse and the ablated volume measurements were within $\pm 15 \%$.

256 laser shots, respectively. This result clearly indicates that non-linear effects occur. These effects are likely due to the gradual formation of the crater with the nonuniform energy radial distribution of the laser (see the Discussion section).

On the same spot of the 2,5-DHB single crystal, the volume of the crater and the ion emission were studied as a function of the laser fluence. In order to get a reliable intensity, MALDI mass spectra were averaged over 256 laser shots. Due to strong saturation effect for the matrix ions at high laser energy, only the ACTH 7-38 peptide signal was studied and recorded. The ion intensity was measured from the total $(\mathrm{M}+\mathrm{H})^{+}$peak area. Figure 6 shows the variation of the ablated volume and that of the peptide ion production as a function of the laser energy. Interestingly, a linear relationship was found for the ablated volume versus the laser energy $\mathrm{E}$ when a classical power law, i.e., (E-Es) ${ }^{4.1}$ with Es corresponding to the ion threshold energy of $3.7 \mu \mathrm{J} /$ pulse, was found for analyte ion production.

\section{Discussion}

\section{Irradiation Effects on 2,5-DHB Single Crystal}

For 2,5-DHB crystals, no specific quenching of the ion production was identified after various irradiation conditions (laser fluence and laser pulse number). Ablation and ion emission are continuously produced as a function of the laser pulse number in all the fluence range. In contrast with sinapinic acid single crystals [9], chemical modification such as decarboxylation of the 2,5DHB crystals irradiated area does not occur or, if it does, the absorption coefficient at the laser wavelength would not be altered. We don't know yet if this strong difference between sinapinic acid and 2,5-DHB can be attributed to the chemical properties of the individual molecule or to the difference in the non-covalent crystal packing. 
An interesting feature of irradiation by a rather low laser pulse number is the observation of ripples at the base of the crater, with a typical periodic length of 5-10 $\mu \mathrm{m}$ (Figure 1) and an orientation parallel to the incident beam direction. Such periodic structures were already found for a large variety of covalent materials (semiconductors, metals, dielectrics...) irradiated by laser pulses over a large range of wavelength (IR and UV), pulse duration (femto- to nanoseconds), and fluence [14-17]. Generally, the ripples are found to be perpendicular to the polarization plane of the laser. Laserinduced periodic surface structures is a well-known phenomenon [15], which is generally interpreted as a cooling down and re-solidification of a molten material under the thermal capillary wave effect [14-18]. The periodic ripples result mainly from the energy relaxation and the thermal fluctuations in the solid, involving a gradient of the surface tension, and they are thus relatively independent of the laser wavelength (nonresonant effect). The ripples found in these studies on 2,5-DHB crystal targets (non-covalent solid) are comparable to those observed on covalent solids (metal and semiconductors) and likely the result of similar mechanisms such as thermal capillary waves [14-17] or Kelvin-Helmhotz instabilities at the interface between the molten layer and the plume [18]. Interestingly, such instabilities would explain the formation of conical microstructures upon laser evaporation of Si [19]. Similar microstructures were also observed on 2,5-DHB single crystals irradiated by UV flat-top laser beam [10].

Of course, the non-homogeneous laser energy distribution in our experiment should play an important role in the local energy absorption and thus in the generation of thermal capillary waves and propagation through a temperature gradient. This can explain that the amplitude of the ripple found in this work is not constant. However, for a rather limited number of laser shots, the ablated volume per shot decreases with an increasing pulse number (Figure 5), i.e., there is a non-linearity between the ablated volume and the laser shots number. This result gives evidence that the periodic structure plays a role in beam absorption and energy relaxation leading to ablation. The gradual formation of the ripple strongly changes the local angle of incidence and thus the reflectivity and the density of absorbed energy in the target. The non-homogeneity of the laser could also reinforce this non-linearity.

For extended irradiation involving a larger number of laser shots, the ripple disappears and the crater is gradually formed as a unique cone oriented along the laser incident direction (Figures 2 and 3). Probably, the gradual ablation prevents the formation of surface instabilities and ripples since the area of the crater surface strongly increases and the energy density absorbed by the matrix strongly varies from one area to another. We have searched for an ablation law compatible with the formation of such a conic crater. We only considered a central slice of matrix composed of 100 elementary surfaces $\mathrm{dS}$ along the plane of the gaussian

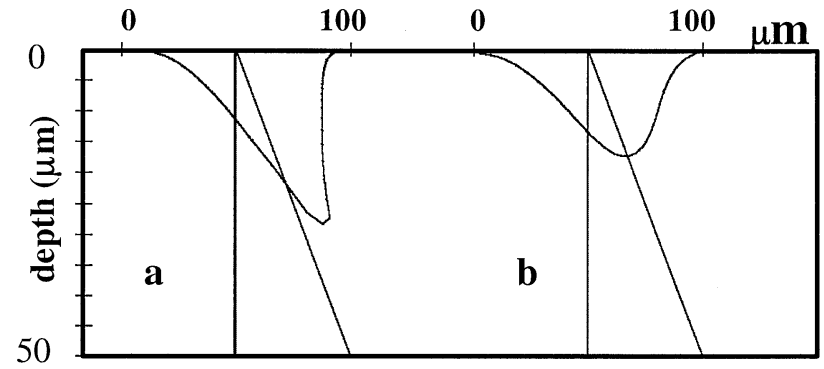

Figure 7. Simulation of the crater formation including a central slice along the plane of laser beam incidence after 1056 laser shots, $2.5 \mu \mathrm{J} /$ pulse. The local ablated depth in $\mu \mathrm{m}$ is $\mathrm{e}_{\mathrm{i}}=\mathrm{A}_{0}\left(\Phi_{\mathrm{i}}-\Phi_{\mathrm{th}}\right)^{n}$ where the ablation threshold fluence $\Phi_{\text {th }}=30 \mathrm{~J} / \mathrm{m}^{2}$, (a) $n=4.5, \mathrm{~A}_{0}$ $=4 \times 10^{-11}$ and gaussian laser profile (FWHM $\left.=40 \mu \mathrm{m}\right)$ and $(\mathbf{b})$ $n=1, \mathrm{~A}_{0}=1 \times 10^{-5}$ and gaussian laser profile $(\mathrm{FWHM}=30 \mu \mathrm{m})$. The oblique line gives the direction of the laser incidence. Note the different scales in radial and depth dimension.

beam incidence. For an element of area $\mathrm{dS}_{\mathrm{i}}$ irradiated with a fluence $\Phi_{\mathrm{i}}$ we have studied the ablated volume as a function of the pulse number using the law: $e_{i}=A_{0}$ $\left(\Phi_{\mathrm{i}}-\mathrm{F}_{\mathrm{th}}\right)^{n}$ where $\mathrm{e}_{\mathrm{i}}$ is the thickness of ablated material, $\Phi_{\text {th }}$ the threshold fluence for ablation, and $\mathrm{A}_{0}$ and $n$ are adjustable parameters. The ablation was intended to be perpendicular to the primary surface $\mathrm{dS}_{\mathrm{i}}$. After one shot, a new angle of incidence is defined by the relative depth of the elements adjacent to $\mathrm{dS}_{\mathrm{i}}$. Simulation of the gradual ablation was performed with a custom-made program (Visual Basic V6.0). Figure 7 shows two typical examples for extended irradiation (1056 laser shots). The general shape of the crater is qualitatively in good agreement with the experimental results (Figure 2) and the coefficient $\mathrm{n}$ can be small $(n=1)$ or large $(n=4.5)$. This shape is determined by the different absorbed energy density depending on the angle of incidence. One should note that for this simple model, the ablated volume per shot from the considered slice decreases very slightly in the range of a few tens shots up to a few thousands shots, in contrast with the experimental results (Figure 5). Of course, these simulations addressed only the central slice of the crater and do not include the entire crater. In addition, for the low irradiation regime $(<256$ laser shots), the ripples are not taken into account, and no reflectivity change with the angle of incidence was considered. Thus, it is difficult to state if the non-linear relationship between the ablated volume and the pulse number is due to a pure geometrical effect (shadow effect, absorption versus angle of incidence. ..) of the crater formation or to other physical phenomena.

\section{Ablated Volume and Ionization}

The striking result in theses studies mainly concerns the difference in the evolution of the ablated volume and of the ion emission as a function of the laser fluence (Figure 6). It has long been recognized that the ablation threshold is lower than that of ion emission for both matrix and analyte $[8,20]$. As shown also in Figure 6, 
the ablation volume is detectable at laser fluence lower than the threshold fluence for peptide ion emission. Simulation of ablation using the breathing-sphere model also showed that the ablated volume (total number of molecules ejected) varies evenly (linear) with the laser fluence [5] just behind the threshold.

The difference in the evolution of ablated volume and ion emission suggests that they likely result from decoupled phenomena. This can be rationalized by considering that the ablation occurs before the end of the laser pulse ( $3 \mathrm{~ns})$ and that a part of the ablated plume also interacts with the laser beam. When the energy absorbed by the matrix is sufficiently high, ablation is induced (vaporization or phase explosion), and the initial plume is irradiated by the rest of the photons. This hypothesis implies that the target does not absorb all the photons in the pulse. Variation of the ablated volume in ceramics as a function of the laser fluence has been already suggested as due to a plasma shielding [16]. This could explain qualitatively why the ablated volume varies linearly with the laser energy or fluence $(\mathrm{V} \sim \Phi)$ and not with the power of the laser energy $\left(\mathrm{V} \sim \Phi^{n}, n>1\right)$. On the other hand, during the short irradiation time $(3 \mathrm{~ns})$, the plume remains very dense (probably its density is close to the solid density) and UV absorption could be, in the first approximation, slightly lower than that of the typical solid. A highly dense matrix plume must include very large clusters and aggregates. The local order in these clusters and aggregates is likely close to that in solid state, and this would promote the thermal-photoionization and energy pooling mechanisms [21, 22]. Matrix ion production would occur in the first stage of ablation and analyte ion production would occur in the expanding plume after desolvation of aggregates [4].

These results support the experiments of two subthreshold photons $[23,24]$ and the recent model proposed by Knochenmuss on the ion generation in MALDI [25], in which the photons of the laser pulse partly interact with the aggregates of the plume in the early stage of its expansion.

\section{Conclusion}

Irradiation effects on large 2,5-DHB crystals were studied over a large fluence range (usual MALDI experiment) and large exposure range. In the low-fluence range and moderate exposures, ripple structures oriented parallel to the incident laser beam direction are the main feature of the crystal surface after ablation. These ripples may be associated with thermal capillary waves generated by the energy relaxation in the solid through the formation on the surface of a molten region. They would also result from surface instabilities during the ablation process.

In the moderate and high fluence range or in the high exposure range, only a crater oriented along the laser beam incident direction is found.

On the same spot of the samples, the analyte ion production and the ablation volume were determined. Interestingly, the ablation volume slowly increases with the laser fluence when the ion production strongly increases by a power-law dependence (exponent $n=$ 4.5). This strong difference suggests first that ablation occurs in a very short time compared to the pulse duration ( $3 \mathrm{~ns})$ and to the ionization process, and second, that photon absorption in the first stage of the plume expansion is particularly efficient to promote ion production.

\section{References}

1. Karas, M.; Hillenkamp, F. Laser Desorption Ionization of Proteins with Molecular Masses Exceeding 10,000 Daltons. Anal. Chem. 1988, 60, 2299-2301.

2. Knochenmuss, R.; Zenobi, R. MALDI Ionization: The Role of In-Plume Processes. Chem. Rev. 2003, 103, 441-452.

3. Karas, M.; Schäfer, J. Ion Formation in MALDI: The Cluster Ionization Mechanism. Chem. Rev. 2003, 103, 427-439.

4. Fournier, I.; Brunot, A; Tabet, J. C;. Int. J. Mass Spectrom. 2002, 213, 203-215.

5. Zhigelei, L. V.; Leveugle, E.; Garrison, B. J.; Yingling, Y. G.; Zeifman, M. Computer Simulations of Laser Ablation of Molecular Substrates. Chem. Rev. 2003, 103, 427-439.

6. Itana, T. E.; Zhigelei, L. V.; Garrison, B. J. Microscopic Mechanisms of Matrix Assisted Laser Desorption of Analyte Molecules: Insights from Molecular Dynamics Simulation. J. Chem. Phys. B 2002, 106, 303-310.

7. Puretzky, A. A;. Phys. Rev. Lett. 1999, 83, 444-447.

8. Dreisewerd, K. The Desorption Process in MALDI. Chem. Rev. 2003, 103, 395-425.

9. Fournier, I.; Tabet, J. C.; Bolbach, G. Irradiation Effects in MALDI and Surface Modifications. Part I: Sinapinic Acid Monocrystals. Int. J. Mass Spectrom. 2002, 219, 515-523.

10. Kampmeier, J.; Dreisewerd, K.; Schürenberg, M.; Strupat, K. Investigations of 2,5-DHB and Succinic Acid as Matrices for IR and UV MALDI. Part I: UV and IR Laser Ablation in the MALDI Process. Int. J. Mass Spectrom. Ion Processes 1997, 169/170, 31-41.

11. Haisa, M.; Kashino, S.; Hanada, S. I.; Tanaka, K.; Okazaki, S.; Shibagaki, M. The Structures of 2-Hydroxy-5-Methylbenzoic Acid and Dimorphs of 2,5-Dihydroxybenzoic Acid. Acta Cryst. B. 1982, 38, 1480-1484.

12. Strupat, K.; Kampmeir, J.; Horneffer, V. Investigations of 2,5-DHB and Succinic Acid as Matrices for UV and IR MALDI. Part II: Crystallographic and Mass Spectrometric Analysis. Int. J. Mass Spectrom. Ion Processes 1997, 169/170, 43-50.

13. Bökelmann, V.; Spengler, B.; Kaufmann, R. Dynamical Parameters of Ion Ejection and Ion Formation in Matrix-Assisted Laser Desorption/Ionization. Eur. Mass Spectrom. 1995, 1, 81-93.

14. Milani, P.; Manfredini, M. Surface Periodic Structures Induced by Pulsed Laser Irradiation of Fullerite. Appl. Phys. Lett. 1996 , 68, 1769.

15. Wang, W. J.; Lu, Y. F.; An, C. W.; Hong, M. H.; Chong, T. C. Controllable Periodic Structures on Silicon Wafer by $\mathrm{CO}_{2}$ Laser Irradiation. Appl. Surf. Sci. 2002, 186, 594-598.

16. Neri, F.; Barreca, F.; Trusso, S. Excimer Laser Ablation of Siliconcarbide Ceramic Targets. Diamond Rel. Mater. 2002, 11, 273-279.

17. Reif, J.; Costache, F.; Henyk, M.; Pandelov, S. Ripples Revisited: Non-Classical Morphology at the Bottom of Femtosecond Laser Ablation Craters in Transparent Dielectrics. Appl. Surf. Sci. 2002, 186, 594-599. 
18. Ang, L. K.; Lau, Y. Y.; Gilgenbach, R. M.; Spindler, H. L.; Lash, J. S.; Kovaleski, S. D. Surface Instabillity of Multipulse Laser Ablation on a Metallic Surface. J. Appl. Phys. 1998, 83, 44664471.

19. Dolgaev, S. I.; Lavrishev, S. V.; Lyalin, A. A.; Simakin, A. V.; Voronov, V. V.; Shafeev, G. A. Formation of Conical Microstructures Upon Laser Evaporation of Solids. Appl. Phys. A 2001, 73, 177-181.

20. Dreisewerd, K.; Schürenberg, M.; Karas, M.; Hillenkamp, F. Influence of the Laser Intensity and Spot Size on the Desorption of Molecules and Ions in Matrix-Assisted Laser Desorptio/Ionization with a Uniform Beam Profile. Int. J. Mass Spectrom. Ion Processes 1995, 141, 127-148.

21. Allwood, D. A.; Dyer, P. E.; Dreyfus, R. W. Ionization Modeling of Matrix Molecules in Ultraviolet Matrix-Assisted Laser
Desorption/Ionization. Rapid Commun. Mass Spectrom. 1997, 11, 499-503.

22. Karbach, V.; Knochenmuss, R. Do Single Matrix Molecules Generate Primary Ions in Ultraviolet Matrix-Assisted Laser Desorption/Ionization? Rapid Commun. Mass Spectrom. 1998, $10,968-974$

23. Tang, X.; Sadeghi, M.; Olumee, Z.; Vertes, A. Matrix-Assisted Laser Desorption/Ionization by Two Cillinear Subthreshold Laser Pulses. Rapid Commun. Mass Spectrom. 1997, 11, 484-488.

24. Knochenmuss, R.; Vertes, A. Time Delayed Two Pulse Studies of MALDI Matrix Ionization Mechanisms. J. Phys. Chem. B 2000, 104, 5406-5410.

25. Knochenmuss, R. A Quantitative Model of Ultraviolet MatrixAssisted Laser Desorption/Ionization. J. Mass Spectrom. 2002, 37, 867-877. 